\title{
Ubi pus, ibi evacua: a review of 601 peritonsillar abscess adult cases
}

\author{
Giorgos Sideris $^{1}\left[\right.$. Vangelis Malamas ${ }^{2} \cdot$ George Tyrellis $^{1} \cdot$ Pavlos Maragkoudakis $^{1} \cdot$ Alexander Delides $^{1}(\mathbb{D}$. \\ Thomas Nikolopoulos ${ }^{1}$
}

Received: 6 September 2021 / Accepted: 26 September 2021 / Published online: 6 October 2021

(c) The Author(s), under exclusive licence to Royal Academy of Medicine in Ireland 2021

\begin{abstract}
Background Peritonsillar abscess (PTA) is the most common deep neck infection, occurring as a consequence of bacterial acute tonsillitis or as a result of infection of the Weber glands, with frequent and life-threatening complications.

Aim To investigate several factors associated with complications and worse prognosis, such as defining the method of surgical drainage and treatment of a PTA which remains an area of controversy in the literature

Methods The purpose of this retrospective study is to examine the epidemiological, clinical, and laboratory findings of 601 adult patients and to discuss them along with their treatment plan.

Results Pharyngalgia was the most common reported symptom, followed by trismus, odynophagia, fever, hot potato voice, malaise, and cervical lymphadenopathy. Sixty-eight patients developed complications. Streptococcus species were the most common pathogens. A statistically significant difference was found in days of hospitalization, WBC and CRP levels, age, and the pre-existing systemic diseases between patients with and without complications. A comparison of patients treated with intravenous and oral antibiotics revealed no statistically significant difference.

Conclusion Over $10 \%$ of PTA cases may develop complications, the most common of which is extension into deep neck spaces. Comorbid conditions increase the risk of complications. Despite the wide range of treatment strategies, incision and drainage remain the cornerstone of surgical treatment. In patients with no comorbidities, intravenous antibiotics appear to have no advantage over oral antibiotics.
\end{abstract}

Keywords Antibiotics $\cdot$ Complications $\cdot$ Deep neck infections $\cdot$ Drainage $\cdot$ Incision $\cdot$ Microbiology $\cdot$ Peritonsillar abscess

\section{Introduction}

Peritonsillar abscess (PTA), also known as quinsy, is a collection of pus between the palatine tonsil and its capsule in the peritonsillar space (PTS). On physical examination, pharyngalgia, odynophagia, a "hot-potato" voice, trismus, fever, malaise, and cervical lymphadenopathy are identified. A unilateral enlargement and a deviated uvula are observed on clinical examination $[1,2]$.

Giorgos Sideris

siderisgior@gmail.com

1 2nd Otolaryngology Department, School of Medicine, "Attikon" University Hospital, National \& Kapodistrian University of Athens, Rimini 1 Chaidari, 12462 Athens, Greece

2 Department of Infomatics, University of Peiraeus, Peiraeus, Greece
Imaging techniques are used to rule out potential problems and aid in the formulation of a final diagnosis. While computed tomography (CT) has a $100 \%$ sensitivity, current studies suggest that transcervical ultrasonography should be used as the first-line diagnostic imaging method [2-4]. PTA is thought to develop in two ways: (a) as a consequence of bacterial acute tonsillitis or (b) as a result of Weber gland infection $[5,6]$.

PTA is frequently caused by a polymicrobial infection [7]. Streptococcus pyogenes, Staphylococcus aureus, Haemophilus influenzae, Prevotella, Porphyromonas, Fusobacterium, and Peptostreptococcus species are the most prevalent pathogens [8]. Treatment strategies include surgical drainage combined with antibiotics against group A streptococcus with anaerobic coverage. PTA is treated surgically using three different techniques: abscess tonsillectomy (ATE), incision and drainage (ID), and needle aspiration (NA). The technique for surgical drainage and therapy continues to be a source of contention in the literature [9-11]. On the other hand, 
other authors report encouraging outcomes when medical treatment is used alone (MTA) [12-14].

Airway obstruction, aspiration of abscess contents, necrosis into the carotid sheath, and subsequent extension into the deep neck spaces or mediastinum are all common and life-threatening complications. A recent assessment assessed the overall mortality rate for individuals with complex PTA to be $10 \%$ [15].

This retrospective study is investigating several epidemiological, clinical, and laboratory factors associated with complications and worse prognosis, such as defining the method of surgical drainage and treatment of a PTA which remains an area of controversy in the literature.

\section{Methods}

This is a retrospective chart review study that has been approved by the Institutional Review Board and ethical committee of the "Attikon" University Hospital — number of approval IRB 204/27-4-20. Reviewed cases were treated at the 2nd Otolaryngology Department from January 2011 to December 2020 with a diagnosis of PTA. PTA was diagnosed based on clinical presentation and initial physical examination. A CT scan was performed in the event that complications or worsening of symptoms were suspected.

Sex, age, comorbidities, symptoms, and days of hospitalization are all evaluated, as are the results of two critical lab tests: white blood cell (WBC) count and C-reactive protein (CRP).

Pharyngalgia, odynophagia, trismus, fever, hot-potato voice, malaise, and cervical lymphadenopathy were all reported as common symptoms. Once referred, all patients receive therapy using one of the following methods: NA+ID or ATE plus intravenous or oral antibiotics. From the time of admission, all patients received a ten-day course of antibiotics. Patients were divided into subgroups to compare clinical and laboratory findings and to determine the efficacy of various treatment strategies.

Mean and standard deviation are used to characterize the parameters. To examine the significance of each independent variable, the ANOVA test was utilized. The 95 percent confidence intervals were used to express the predicted accuracy (CIs). P 0.05 was used as the criterion of significance. IBM SPSS Statistics, version 19, was used for statistical analysis.

\section{Results}

Six hundred one adult patients (386 males, 215 females) were included in the study. Mean age was $38.5 \pm 14.5$ years (95\% CI: 37.4-40). The mean total WBC count was $13.986 \pm 3.782 \times 10^{3} / \mu \mathrm{L}(95 \% \mathrm{CI}: 13.683-14.289)$, and the mean CRP level was $82 \pm 65.6 \mathrm{mg} / \mathrm{L}$ (95\% CI: 76.7-87.2). Pharyngalgia was the most common reported symptom, followed by trismus, odynophagia, fever, hot-potato voice, malaise, and cervical lymphadenopathy. Prior to admission, 82 patients were receiving oral antibiotics. A CTscan was performed on 112 patients. Comorbidities were present in 242 patients while 24 of them had two or more comorbidities (cardiovascular diseases: 122 patients, diabetes mellitus: 65 , respiratory diseases: 37 , gastrointestinal disorders: 10, endocrine disorders: 10 , chronic renal failure: 7 , hematologic malignancies: 5 , rheumatoid arthritis: 4, psychiatric disorders: 4, HIV: 2). Five hundred sixtyone patients were treated with NA + ID and intravenous antibiotics, while 12 patients underwent an abscess tonsillectomy due to an intratonsillar abscess or recurrence of PTA (inpatient - IP - Group). 28 patients were treated as outpatients with NA + ID plus, oral antibiotics (outpatient - OP - Group).

Four hundred two inpatients were treated with iv penicillin, 103 with iv penicillin plus medronidazole and 68 with iv clindamycin. Twenty-eight patients that were treated as outpatients received oral amoxicillin plus clavulanic acid. Sixty-eight patients developed or progressed to PTA complications in total. Parapharyngeal abscess (35 adult patients) was the most common complication followed by salivary gland abscess (9), acute epiglottitis (6), retropharyngeal abscess (4), necrotizing fasciitis (4), hemorrhage (4), masticator space abscess (3), and multiple space abscess (3).

Table 1 Patients' clinical profile, treatment, and outcome

\begin{tabular}{lll}
\hline & & Adults $(n=601)$ \\
\hline Demographics & Age (years) & 38.5 \\
& Male & 386 \\
Clinical signs & Female & 215 \\
& Pharyngalgia & 556 \\
& Odynophagia & 322 \\
& Hot-potato voice & 112 \\
& Trismus & 365 \\
& Fever & 195 \\
Laboratory findings & Malaise & 178 \\
Comorbidities & Lymphadenopathy & 92 \\
Treatment & WBC $\left(\times 10^{3} / \mu \mathrm{L}\right)$ & $13.986 \pm 3.782$ \\
& CRP $($ mg/L) & $82 \pm 65.6$ \\
& & 242 \\
Complications & ID + iv antibiotics & 561 \\
Outcome & ATE & 28 \\
& MTA & 12 \\
& & 0 \\
& Survive & 68 \\
& Death & 600 \\
& & 1 \\
& & \\
& &
\end{tabular}


Table 2 Microbiological findings

\begin{tabular}{ll}
\hline Pathogen & $\begin{array}{l}\text { No. of } \\
\text { patients } \\
(n=280)\end{array}$ \\
\hline Streptococcus pneumoniae & 11 \\
Streptococcus viridans & 13 \\
Group A streptococcus & 78 \\
Group B streptococcus & 32 \\
Staphylococcus species & 16 \\
Fusobacterium necrophorum & 27 \\
Anaerobic bacteria & 35 \\
Others & 21 \\
Mixed flora & 47 \\
\hline
\end{tabular}

Six hundred thirty-four patients survived while one (1) died (Table 1).

A total of 352 pus cultures were collected with 280 of them isolating microorganisms. Streptococcus species were the most common pathogens accounting for $47.9 \%$ of the total sample with Group A streptococcus isolated from 78 cultures. In 47 cultures, multiple pathogens were isolated (Table 2).

A statistical significant difference was found in days of hospitalization, WBC and CRP levels, age, and the preexistence of systemic diseases between inpatients with and without complications (Table 3).

There was no statistically significant difference in age, complications, blood inflammatory markers, or comorbidities between patients treated with ID plus IV antibiotics and those treated as outpatients (Table 4).

\section{Discussion}

PTA remains the most common deep neck infection, usually seen in young to middle-aged individuals $[1,5]$. Our statistics confirm this conclusion, as the mean age of our patients was 38.5 years.
As expected, pharyngalgia, odynophagia, and trismus were the most common reported symptoms, corroborated by the literature $[1,3,16]$. Regardless of its frequency, trismus continues to be a "gray" pathognomonic indication. PTA is a collection of pus positioned between the tonsillar capsule and the pharyngeal constrictor muscle, while the masseter, temporalis, medial pterygoid, lateral pterygoid, digastric, and hyoid muscles are responsible for opening and closing the mouth. Thus, it is important to keep in mind that trismus may signal a parapharyngeal abscess even in the absence of a concurrent PTA [17]. Our findings indicate that trismus is statistically insignificant when comparing inpatient and outpatient adults (IP: 349, OP: 16, $p$-value: 0.56 ), as well as when comparing inpatient adults with and without comorbidities (with complications: 44, without complications: 305, p-value: 0.769).

Numerous studies have established a link between the prevalence and severity of PTA and comorbidities. According to Martínez Pascual et al., systemic comorbidities are associated with a worse prognosis in patients undergoing PTA [16]. Wu et al. found a link between diabetes mellitus type 2 and an increased risk of PTA [18]. Ding et al. note that PTA is more prevalent in patients with rheumatoid arthritis during 5 years of diagnosis [19]. Our data indicate that individuals with comorbidities are at an increased risk of developing complications; hence, they should be treated as inpatients, and oral antibiotics should be chosen for adults without systemic disorders. Cereceda-Monteoliva et al. confirm this observation, proposing that, particularly in the COVID-19 era, PTA care should be offered to patients without systematic comorbidities in an outpatient setting [20].

Our data support the first-line use of penicillin, as streptococcus species were the most common isolated microorganisms among 280 positive culture results. Hallgren et al. discovered no significant differences in recovery and recurrence rates between patients treated with penicillin and those treated with clindamycin [21]. Thirty-seven cultures identified anaerobic pathogens, and 47 cultures isolated mixed flora in our series. This finding implies that routine

Table 3 Comparison between inpatients (IP) with and without complications

\begin{tabular}{|c|c|c|c|c|}
\hline & & IP Group No complications $(n=509)$ & IP Group With complications $(n=64)$ & p-value \\
\hline \multirow[t]{2}{*}{ Age } & Mean $\pm S D$ & $37.9 \pm 14.5$ (min: $17, \max : 87)$ & $45.4 \pm 15.5$ (min: $17, \max 86)$ & \multirow[t]{2}{*}{$<0.00$} \\
\hline & $95 \% \mathrm{CI}$ & $36.6-39.1$ & $41.6-49.3$ & \\
\hline Comorbidities & & 195 & 42 & $<0.001$ \\
\hline \multirow[t]{2}{*}{ WBC } & Mean $\pm S D$ & $13,779 \pm 3690$ & $15,395 \pm 4450$ & \multirow[t]{2}{*}{$<0.00$} \\
\hline & $95 \% \mathrm{CI}$ & $13,456-14,100$ & $14,284-16,507$ & \\
\hline \multirow[t]{2}{*}{ CRP } & Mean $\pm S D$ & $77.6 \pm 60.8$ & $125.5 \pm 90$ & \multirow[t]{2}{*}{$<0.00$} \\
\hline & $95 \% \mathrm{CI}$ & $72.2-82.8$ & $103-148$ & \\
\hline \multirow[t]{2}{*}{ Hospitalization (days) } & Mean $\pm S D$ & $2.96 \pm 1.7(\min : 1, \max : 7)$ & $6.53 \pm 4.26(\min : 3, \max : 21)$ & \multirow[t]{2}{*}{$<0.00$} \\
\hline & $95 \% \mathrm{CI}$ & $2.81-3.11$ & $5.47-7.59$ & \\
\hline
\end{tabular}


Table 4 Comparison between patients that received iv (IP) and oral (OP) antibiotics

\begin{tabular}{lllll}
\hline & & IP Group $(n=573)$ & OP Group $(n=28)$ & p-value \\
\hline Age & Mean $\pm S D$ & $38.7 \pm 14.7$ & $34.8 \pm 8.4$ & 0.16 \\
Comorbidities & $95 \%$ CI & $37.5-39.9$ & $31.52-38$ & \\
WBC & & 237 & 5 & 0.013 \\
& Mean $\pm S D$ & $14,960 \pm 3812$ & $14,526 \pm 3118$ & 0.44 \\
CRP & $95 \%$ CI & $13,647-14,272$ & $13,318-15,735$ & \\
& Mean $\pm S D$ & $82.8 \pm 66.4$ & $64.1 \pm 42.8$ & 0.14 \\
Complications & $95 \%$ CI & $77.4-88.3$ & $47.5-80.7$ & \\
\hline
\end{tabular}

antibiotic treatment should include anaerobe pathogens [22, 23].

Several comparative studies between surgical and nonsurgical therapy approaches, such as ATE, ID, NA, and MTA, have produced conflicting outcomes. Hahn et al. support the notion that ATE in an inpatient setting is a relatively safe and effective primary therapeutic choice, whereas ID is a more desirable alternative for patients without comorbidities [9]. According to Zebolsky et al., MTA may be a safe and successful alternative to surgical drainage for the empiric therapy of PTA, whereas Urban et al. describe comparable findings $[12,13]$. In both of the aforementioned studies, the size of the abscess did not appear to have an effect on treatment failure. Forner et al. found no difference in the likelihood of treatment failure between individuals managed with MTA and those managed surgically, but cautioned that these data should be interpreted cautiously due to a high probability of bias and overall low quality of research [24]. Chang et al. find that very limited evidence suggests that ID may be associated with a lower risk of recurrence than NA [10]. On the contrary, in their study, Mansour et al. showed a significant reduction in the length of hospitalization for PTA patients treated with ID over NA, as well as a decreased chance of repeating the surgery [11]. Battaglia et al. demonstrate that MTA appears to be as safe and effective as surgical drainage, with the added benefit of reduced pain and a shorter length of hospitalization, particularly in patients without trismus [14].

According to our institutional recommendations, NA + ID is the most advantageous surgical technique for PTA. The fact that 82 of our patients were getting oral antibiotics previous to admission and symptoms resolved only after ND+ ID reminds us of the phrase "ubi pus, ibi evacua," which is still somewhat applicable today. It is the authors' opinion that ATE should be considered in patients with recurrent or intratonsillar PTA to prevent the complications associated with a standard operation performed under general anesthesia, while NA appears to lengthen hospitalization due to the requirement for numerous repeats. Additionally, the authors emphasize the importance of a surgeon always paying attention to the ID technique. According to a recent study conducted by Tasli et al., the mean distance between the anterior border of the PTA and the internal carotid artery is $3.6 \mathrm{~cm} \mathrm{[25].} \mathrm{Although} \mathrm{hemorrhage} \mathrm{was} \mathrm{found} \mathrm{during} \mathrm{ID}$ in four of our patients, this was not due to an injury to the internal carotid artery but to another vascular.

Klug et al. observed that the most often described consequences were descending mediastinitis, parapharyngeal and retropharyngeal abscess, necrotizing fasciitis, and Lemierre's syndrome [15]. Males and patients over the age of 40 appear to be at an elevated risk of developing severe disease in the same study, while the majority of patients presented with concurrent PTA and its complications. Our data confirm this, indicating that PTA patients over the age of 45, as well as those with a WBC count greater than $15.000 \times 10^{3}$ $/ \mathrm{L}$ and CRP levels greater than $110 \mathrm{mg} / \mathrm{L}$, are more likely to suffer complications. The most often encountered complication in our investigation was parapharyngeal abscess. This is unsurprising given that it is a "classic route" for the spread of PTA infection [17]. PTA extension into salivary gland regions was the second most often encountered problem ( 7 patients). Kaltiainen et al. find that small salivary glands were present in 77 (67.5\%) of 114 adult tonsils following initial histological evaluation [26]. Our findings are consistent with the idea that small salivary glands play a role in PTA formation [26, 27].

\section{Conclusion}

PTA remains a potentially life-threatening medical condition, with over $10 \%$ of patients developing complications, the most common of which is extension into deep neck spaces. Patients with comorbidities require a longer hospital stay and are more prone to experience complications. Despite the variety of treatment techniques available, our findings demonstrate that incision and drainage remain the cornerstone of safe and effective surgical treatment. In patients without comorbidities, intravenous antibiotics appear to have no advantage over oral antibiotics. 
Availability of data and material Not applicable.

Code availability Not applicable.

\section{Declarations}

Ethical approval All procedures performed in studies involving human participants were in accordance with the ethical standards of the institutional research committee and with the 1964 Helsinki Declaration and its later amendments or comparable ethical standards.

Informed consent Informed consent was obtained from all individual participants included in the study.

Conflict of interest The authors declare no competing interests.

\section{References}

1. Li RM, Kiemeney M (2019) Infections of the neck. Emerg Med Clin North Am 37(1):95-107. https://doi.org/10.1016/j.emc.2018.09.003

2. Klein MR (2019) Infections of the oropharynx. Emerg Med Clin North Am 37(1):69-80. https://doi.org/10.1016/j.emc.2018.09.002

3. Scott PM, Loftus WK, Kew J et al (1999) Diagnosis of peritonsillar infections: a prospective study of ultrasound, computerized tomography and clinical diagnosis. J Laryngol Otol 113(3):229-232. https://doi.org/10.1017/s0022215100143634

4. Sievert M, Miksch M, Mantsopoulos K et al (2021) The value of transcutaneous ultrasound in the diagnosis of tonsillar abscess: a retrospective analysis [published online ahead of print, $2021 \mathrm{Apr}$ 24]. Auris Nasus Larynx S0385-8146(21):00127-9. https://doi.org/ 10.1016/j.anl.2021.04.006

5. Stage J, Bonding P (1987) Peritonsillar abscess with parapharyngeal involvement: incidence and treatment. Clin Otolaryngol Allied Sci 12(1):1-5. https://doi.org/10.1111/j.1365-2273.1987.tb00155.x

6. Kordeluk S, Novack L, Puterman M et al (2011) Relation between peritonsillar infection and acute tonsillitis: myth or reality? Otolaryngol Head Neck Surg 145(6):940-945. https://doi.org/10.1177/ 0194599811415802

7. Galioto NJ (2017) Peritonsillar abscess. Am Fam Physician 95(8):501-506

8. Brook I (2004) Microbiology and management of peritonsillar, retropharyngeal, and parapharyngeal abscesses. J Oral Maxillofac Surg 62(12):1545-1550. https://doi.org/10.1016/j.joms.2003.12.043

9. Hahn J, Barth I, Wigand MC et al (2021) The surgical treatment of peritonsillar abscess: a retrospective analysis in 584 patients [published online ahead of print, 2021 Jun 10]. Laryngoscope. https:// doi.org/10.1002/lary.29677

10. Chang BA, Thamboo A, Burton MJ (2016) Needle aspiration versus incision and drainage for the treatment of peritonsillar abscess. Cochrane Database Syst Rev 12(12):CD006287. Published 2016 Dec 23. https://doi.org/10.1002/14651858.CD006287.pub4

11. Mansour C, De Bonnecaze G, Mouchon E et al (2019) Comparison of needle aspiration versus incision and drainage under local anaesthesia for the initial treatment of peritonsillar abscess. Eur Arch Otorhinolaryngol 276(9):2595-2601. https://doi.org/10.1007/ s00405-019-05542-1

12. Zebolsky AL, Dewey J, Swayze EJ et al (2021) Empiric treatment for peritonsillar abscess: a single-center experience with medical therapy alone. Am J Otolaryngol 42(4):102954. https://doi.org/10. 1016/j.amjoto.2021.102954
13. Urban MJ, Masliah J, Heyd C et al (2021) Peritonsillar abscess size as a predictor of medical therapy success [published online ahead of print, 2021 May 12]. Ann Otol Rhinol Laryngol 34894211015590. https://doi.org/10.1177/00034894211015590

14. Battaglia A, Burchette R, Hussman J et al (2018) Comparison of medical therapy alone to medical therapy with surgical treatment of peritonsillar abscess. Otolaryngol Head Neck Surg 158(2):280-286. https://doi.org/10.1177/0194599817739277

15. Klug TE, Greve T, Hentze M (2020) Complications of peritonsillar abscess. Ann Clin Microbiol Antimicrob 19(1):32. Published 2020 Jul 30. https://doi.org/10.1186/s12941-020-00375-x

16. Martínez Pascual P, Pinacho Martinez P, Friedlander E et al (2018) Peritonsillar and deep neck infections: a review of 330 cases. Braz J Otorhinolaryngol 84(3):305-310. https://doi.org/10.1016/j.bjorl. 2017.03.008

17. Klug TE, Fischer AS, Antonsen C et al (2014) Parapharyngeal abscess is frequently associated with concomitant peritonsillar abscess. Eur Arch Otorhinolaryngol 271(6):1701-1707. https://doi. org/10.1007/s00405-013-2667-x

18. Wu CL, Tsai MS, Lee TJ et al (2021) Type 2 diabetes mellitus increases peritonsillar abscess susceptibility: real-world evidence [published online ahead of print, 2021 Jan 14]. Clin Exp Otorhinolaryngol. https://doi.org/10.21053/ceo.2020.02257

19. Ding MC, Tsai MS, Yang YH et al (2021) Patients with comorbid rheumatoid arthritis are predisposed to peritonsillar abscess: real-world evidence [published online ahead of print, 2021 Feb 3]. Eur Arch Otorhinolaryngol. https://doi.org/10.1007/ s00405-021-06638-3

20. Cereceda-Monteoliva N, Devabalan Y, Lorenz H et al (2021) Improving the management of suspected tonsillitis and peritonsillar abscess referred to ENT - a coronavirus disease 2019 service improvement. J Laryngol Otol 135(7):584-588. https://doi.org/10.1017/ S0022215121001213

21. Hallgren F, Lindell E, Nilsson-Helger B, Lundqvist A (2021) Antibiotics in treatment of peritonsillar infection: clindamycin versus penicillin. J Laryngol Otol 135(1):64-69. https://doi.org/10.1017/ S002221512100013X

22. Herzon FS, Meiklejohn DA, Hobbs EA (2018) What antibiotic should be used in the management of an otherwise healthy adult with a peritonsillar abscess? Laryngoscope 128(4):783-784. https:// doi.org/10.1002/lary.26881

23. Johnston J, Stretton M, Mahadevan M, Douglas RG (2018) Peritonsillar abscess: a retrospective case series of 1773 patients. Clin Otolaryngol 43(3):940-944. https://doi.org/10.1111/coa.13070

24. Forner D, Curry DE, Hancock K et al (2020) Medical intervention alone vs surgical drainage for treatment of peritonsillar abscess: a systematic review and meta-analysis. Otolaryngol Head Neck Surg 163(5):915-922. https://doi.org/10.1177/0194599820927328

25. Tasli H, Ozen A, Akca ME, Karakoc O (2020) Risk of internal carotid injury due to peritonsillar abscess drainage. Auris Nasus Larynx 47(6):1027-1032. https://doi.org/10.1016/j.anl.2020.06.001

26. Kaltiainen E, Wikstén J, Aaltonen LM et al (2017) The presence of minor salivary glands in the peritonsillar space. Eur Arch Otorhinolaryngol 274(11):3997-4001. https://doi.org/10.1007/ s00405-017-4738-x

27. Sanmark E, Wikstén J, Välimaa $\mathrm{H}$ et al (2020) Peritonsillar abscess may not always be a complication of acute tonsillitis: a prospective cohort study. PLoS One 15(4):e0228122. Published $2020 \mathrm{Apr}$ 3. https://doi.org/10.1371/journal.pone.0228122

Publisher's Note Springer Nature remains neutral with regard to jurisdictional claims in published maps and institutional affiliations. 